\title{
Strain-induced grain refinement of cobalt during surface mechanical attrition treatment
}

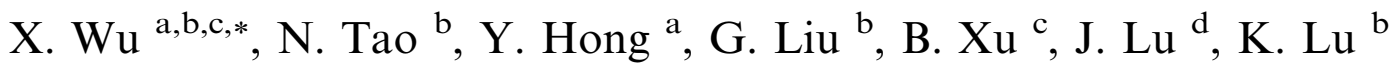 \\ ${ }^{a}$ State Key Laboratory of Nonlinear Mechanics, Institute of Mechanics, Chinese Academy of Sciences, Beijing 100080, China \\ b Shenyang National Laboratory for Materials Sciences, Institute of Metal Research, Chinese Academy of Sciences, Shenyang 110016, China \\ ${ }^{\mathrm{c}}$ College of Materials Science and Technology, Taiyuan University of Technology, Taiyuan 030024, China \\ ${ }^{\mathrm{d}}$ LASMIS, University of Technical of Troyes, 10000 Troyes, France
}

Received 21 September 2004; received in revised form 5 October 2004; accepted 12 October 2004

Available online 11 November 2004

\begin{abstract}
The microstructural evolution during surface mechanical attrition treatment of cobalt (a mixture of hexagonal close packed (hcp) and face-centered cubic (fcc) phases) was investigated, in order to reveal the mechanism of grain refinement and strain accommodation. The microstructure was systematically characterized by both cross-sectional and planar-view transmission electron microscopy. In the hcp phase, the process of grain refinement, accompanied by an increase in strain imposed in the surface layer, involved: (1) the onset of $\left\{\begin{array}{lll}0 & \overline{1} & 1\end{array}\right\}$ deformation twinning, (2) the operation of $\langle 11 \overline{2} 0\rangle\{10 \overline{1} 0\}$ prismatic and $\langle 11 \overline{2} 0\rangle(0001)$ basal slip, leading to the formation of low-angle dislocation boundaries, and (3) the successive subdivision of grains to a finer and finer scale, resulting in the formation of highly misoriented nanocrystalline grains. Moreover, the formation of nanocrystallites at the grain boundary and triple junction was also observed to occur concurrently with straining. By contrast, the fcc phase accommodated strain in a sequence as follows: (1) slip of dislocations by forming intersecting planar arrays of dislocations, (2) \{111\} deformation twinning, and (3) the $\gamma(\mathrm{fcc}) \rightarrow \varepsilon(\mathrm{hcp})$ martensitic phase transformation. The mechanism of grain refinement was interpreted in terms of the structural subdivision of grains together with dynamic recrystallization occurring in the hcp phase and the $\gamma \rightarrow \varepsilon$ martensitic transformation in the fec phase as well.
\end{abstract}

(C) 2004 Acta Materialia Inc. Published by Elsevier Ltd. All rights reserved.

Keywords: Nanostructure; Plastic deformation; Martensitic transformation; Surface mechanical attrition treatment; Cobalt

\section{Introduction}

One exciting benefit of plastically deforming metallic materials to ultra-large strains and/or high strain rates is the significant refinement of grain sizes down to the nanometer level. Hence, a better understanding of the fundamental mechanism of straining-induced grain refinement is of academic and technological significance. This is because the nanocrystalline (nc, grain size $d<100 \mathrm{~nm}$ ) metals and alloys often possess unusual

\footnotetext{
${ }^{*}$ Corresponding author. Tel.: +86 10 62618150, fax: +86 10 62561284

E-mail address: xlwu@imech.ac.cn (X. Wu).
}

mechanical properties which stem mainly from the much reduced grain size and resultant unique deformation mechanism [1-3]; in addition, grain refinement is crucial for development of synthesis techniques that are oriented towards practical applications for engineering materials $[4,5]$.

So far, most documented experimental and theoretical investigations in the literature [6-10] show that the grain refinement is originated from dislocation activities during deformation in cubic (face-centered cubic (fcc) and body-centered cubic (bcc) as well) metals and alloys with a medium to high stacking fault energy (SFE). Plastic straining generates high density dislocations arranged into various configurations depending on the nature of 
materials, such as the geometrically necessary boundary, incidental dislocation boundary, and dense dislocation wall, etc. [6-8]. These dislocation boundaries increase their misorientations with increasing strain, and some of them will become high angle ones, which subdivide the original grains down to the refined grain sizes. By contrast, the low SFE fcc materials exhibit a different mode of grain refinement. In an Inconel 600 alloy [11] and AISI 304 stainless steel [12] (both have low SFEs), for example, the grain refinement involves the slip of dislocations and subsequent deformation twinning, followed by interplay of twins with dislocations. As for the hexagonal close packed (hcp) metals, deformation twinning occurs at the early stage of deformation and serves as an additional deformation mechanism to dislocation slip in order to satisfy the von Mises criterion. The transition of strain accommodation from twinning to dislocation slip with increasing strain is responsible for the grain refinement in titanium [13,14], magnesium [15], and zirconium [16] during the process of equalchannel angular pressing (ECAP).

Pure cobalt, with an hep crystal structure at room temperature, has a very low SFE $\left(27 \pm 4 \mathrm{~mJ} \mathrm{~m}^{-2}\right)$ [17] compared with titanium $\left(>300 \mathrm{~mJ} \mathrm{~m}^{-2}\right)$, zirconium $\left(80 \mathrm{~mJ} \mathrm{~m}^{-2}\right)$ and magnesium $\left(50-80 \mathrm{~mJ} \mathrm{~m}^{-2}\right)$. Cobalt has a martensitic phase transformation occurring at about $690 \mathrm{~K}$ from the high temperature $\gamma$ (fcc) phase to low temperature $\varepsilon$ (hcp) phase [18]. The $\gamma \rightarrow \varepsilon$ transformation is achieved by glide of transformation dislocations (i.e., partials) on every other close packed plane. However, this transformation is very sluggish and does not occur merely by cooling below lower transformation temperature. For instance, quenching from the high temperature phase retained up to $30 \%$ fcc phase which then transformed on plastic deformation by shear-activated martensitic transformation and the extent depends on the amount of deformation imposed [19]. The $\gamma \rightarrow \varepsilon$ transformation causes mainly the partials and stacking faults (SFs) inside the hop phase [20]. Three kinds of basal SFs may occur in cobalt: two intrinsic faults $\left(I_{1}\right.$ and $\left.I_{2}\right)$ and one extrinsic fault $(E)$. The Burgers vectors of the partials surrounding them are of the type $\mathbf{p}+\mathbf{c} / 2\langle 2 \overline{2} 03\rangle, \mathbf{p}=1 / 3\langle 1 \overline{1} 00\rangle$ and $\mathbf{c} / 2\langle 0001\rangle$ in the case of $I_{1}, I_{2}$ and $E$, respectively. $I_{1}$ and $E$ faults are formed during the phase transformation. Only the fault $I_{2}$ can be produced directly by shear in such a way that the dislocations gliding on the basal planes dissociate into Shockley partials bounding an intrinsic SF of type $I_{2}$ [20].

The prevailing slip system is the $\langle 11 \overline{2} 0\rangle(0001)$ basal slip during plastic deformation of cobalt $[21,22]$. In contrast, prolific deformation twinning may occur on various planes, e.g., $\{10 \overline{1} 2\},\{11 \overline{2} 2\}$, and $\{11 \overline{2} 1\}$, but most of them were observed in single crystals $[22,23]$. Recently, Sort et al. [24] have found that large amounts of SFs, especially deformation faults, are created in ball milled nc cobalt powders during high pressure torsion. $\mathrm{X}$-ray diffraction has shown the presence of $\gamma \rightarrow \varepsilon$ transformation during ball milling of nc cobalt powders [25]. Especially, nc cobalt exhibited unusual mechanical properties such as remarkable work hardening and ductility comparable to those in coarse-grained cobalt [26].

From a scientific point-of-view, cobalt has a low SFE and experiences $\gamma \rightarrow \varepsilon$ transformation during deformation; developing an understanding of the origin and mechanism of grain refinement during plastic straining has intrinsic merit. For this purpose, pure cobalt (a mixture of hcp and fcc phases) was plastically deformed using the surface mechanical attrition treatment (SMAT) [27]. The microstructure change was determined to establish the mechanism of grain refinement and strain accommodation during SMAT in order to provide insight into optimal methods for grain refinement and the improvement of mechanical properties.

\section{Experimental}

The pure cobalt (purity wt $\% 99.98$ ) used in the present investigation was electrodeposited in a plate shape. The average grain size was determined to be $\sim 30 \mu \mathrm{m}$. The X-ray diffraction analysis indicated a duplex structure of the product consisting of the hcp and fcc (volume fraction $\sim 18 \%$ ) phases. In fact, it is difficult to obtain polycrystalline cobalt with $100 \%$ hcp phase, and some amount of metastable fcc phase always remains at room temperature regardless of the applied treatment [19,28]. The plate was mechanically peeled off to a dimension of $5 \times 40 \times 100 \mathrm{~mm}$ suitable to SMAT processing.

A detailed description of the SMAT apparatus used in this study was reported previously $[8,27]$. In brief, during the SMAT process, the hardened steel balls (shots) of $8 \mathrm{~mm}$ in diameter were placed at the bottom of a cylinder-shaped vacuum chamber attached to a vibration generator, with which the balls were resonated. Because of the high vibration frequency of the system, the sample surface to be treated was peened repetitively by a large number of balls within a short period of time. Each peening of the ball to the surface resulted in plastic deformation in the surface layer of the treated sample. As a consequence the grains in the surface layer were expected to be effectively refined. In the present work, the SMAT process was performed for $50 \mathrm{~min}$ at room temperature with a vibrating frequency of $50 \mathrm{~Hz}$ under vacuum. As for the deformed material, both strain and strain rate had a gradient variation from the treated surface (both are extremely large) towards the deep matrix (essentially zero), and as a result, the grain sizes with gradient distribution were developed in the treated sample $[8,9,27]$. Hence, one may examine the microstructure characteristics at different levels of strain to reveal the underlying mecha- 
nism of grain refinement within various grain size regimes. In addition, during the process of SMAT, Fe may contaminate the treated surface layer from peening balls as often happened in mechanical attrition/milling process. The present investigation focused solely on the layer of pure cobalt without Fe contamination.

Following SMAT, the microstructure characterization was performed in a transmission electron microscope (TEM, JEM200CX) operated at $200 \mathrm{kV}$. Both cross-sectional and planar-view thin foils for TEM observation were prepared. The cross-sectional foils were prepared as follows: (1) two pieces $(1 \times 2 \times 5 \mathrm{~mm}$ in size) of the treated layer were cut off and bonded face to face together using a glue; (2) this was put into a die to press and hold for $24 \mathrm{~h}$; (3) it was then sliced into thin sheets of about $0.1 \mathrm{~mm}$ thick normal to the treated surface; and (4) sheets were then mechanically thinned down to about $40 \mu \mathrm{m}$ thick followed by Ar ion milling near the bonding line using a Gatan PIPS with small incident angle, allowing large, thin and homogeneous areas to be obtained. The planar-view TEM foils of deformed layers at different depths were obtained first by polishing the corresponding surface layer, then electrochemically thinning the samples on two sides in a solution of equal volumes of $\mathrm{H}_{3} \mathrm{PO}_{4}$ and $30 \% \mathrm{H}_{2} \mathrm{O}_{2}$ at $\sim 0{ }^{\circ} \mathrm{C}$ until it was about $40 \mu \mathrm{m}$ thick; they were finally electro-polished using a twin-jet technique in a solution of $10 \%$ perchloric acid and $90 \%$ 2-butoxyethnal at a voltage of $30 \mathrm{~V}$ and a temperature of $5{ }^{\circ} \mathrm{C}$.

\section{Experimental results}

\subsection{0-180 $\mu m$ below treated surface}

\subsection{1. hep phase}

Fig. 1(a) is a cross-sectional bright-field TEM micrograph of the microstructure at about $170 \mu \mathrm{m}$ deep below treated surface. The nearly parallel plates with varying widths are visible. Fig. 1(b) is the selected-area electron diffraction (SAED) pattern taken using a [ $\left[\begin{array}{lll}1 & 1 & 1\end{array}\right]$ zone axis from the region containing two adjacent plates labeled $\mathrm{A}$ and $\mathrm{B}$. The mirror spots appear with respect to the (10)11) plane, indicating that two plates constituted a $\{10 \overline{1} 1\}$ twin structure, but two $\{10 \overline{1} 1\}$ twin planes slightly misaligned by $\sim 10^{\circ}$. The presence of SFs lying on $\{0001\}$ basal planes is also visible inside the adjacent plates labeled B and C. This indicates concurrent dislocation behaviors accompanying twinning deformation. Although the gross twinning shear due to $\{10 \overline{1} 1\}$ twinning is quite small [29], the change in crystallographic orientation is such that the $(0001)$ plane of the twin is rotated $\sim 71^{\circ}$ from that of the untwined portion. The same twin system was consistently observed for more than 30 different hcp grains at various depths ranging from $\sim 130$ to $180 \mu \mathrm{m}$ adjacent to the strain-free matrix.
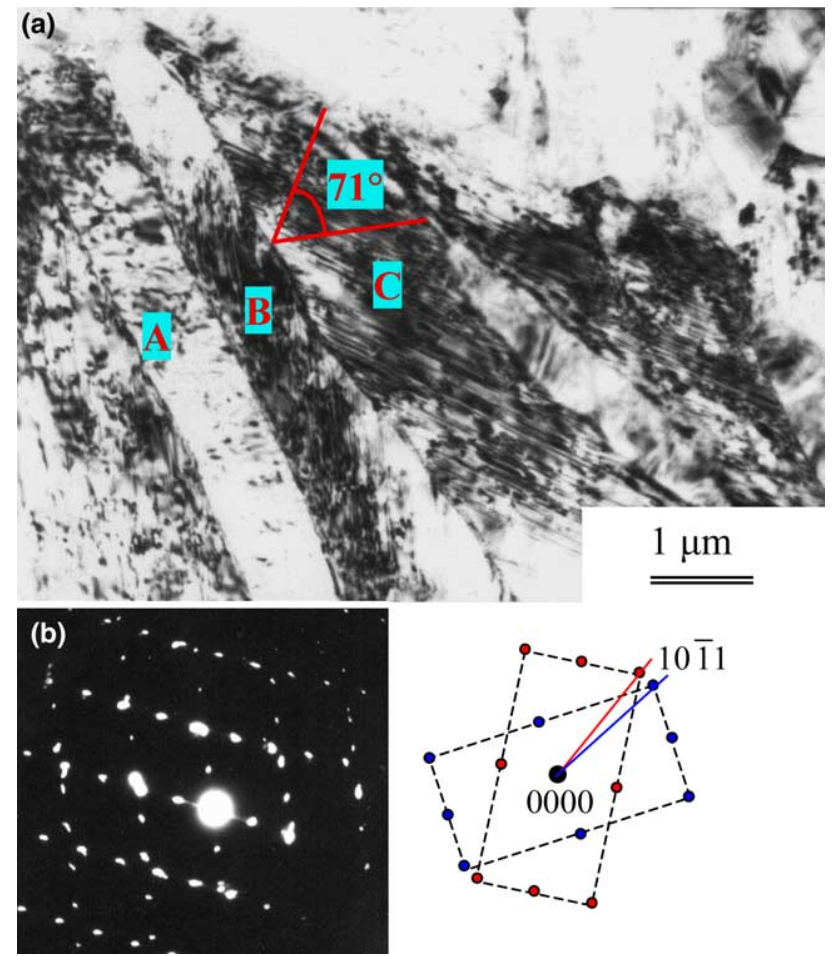

Fig. 1. (a) TEM micrograph showing the deformation twins in the hcp grain $(\sim 170 \mu \mathrm{m}$ deep below treated surface); (b) selected-area electron

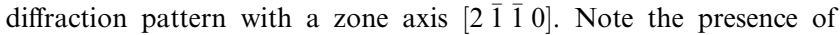
stacking faults in the interior of twin platelets.

Therefore, $\{10 \overline{1} 1\}$ deformation twinning is determined to be the main mode of accommodating strain at the low strain level during the SMAT process.

The deformation-induced $(0001)$ basal SFs of high density were extensively observed to exist in the interiors of hcp grains as seen in Fig. $2(\sim 170 \mu \mathrm{m}$ below treated surface). In most cases the SFs span through the entire grain. The formation of high density SFs is probably

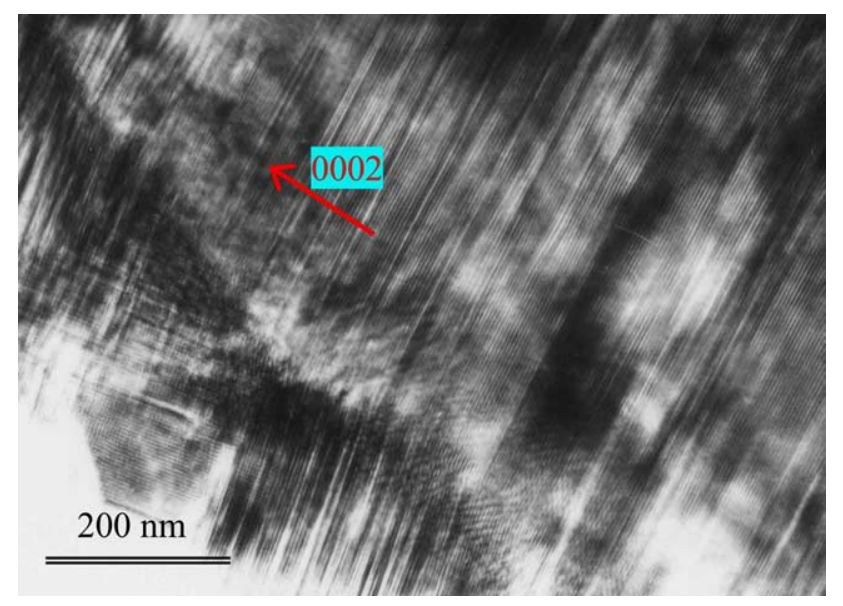

Fig. 2. The (0001) basal stacking faults in the hep grain $(\sim 170 \mu \mathrm{m}$ deep). 
due to the successive formation of SFs at various basal planes with strain.

In order to analyze the characteristics of dislocations of the hcp grain, the TEM images were taken under twobeam diffraction conditions using different diffraction vectors. Thin foils were tilted in such a way that the incident beam direction was aligned parallel to either $\left[\begin{array}{lll}0 & 1 & 1\end{array}\right]$

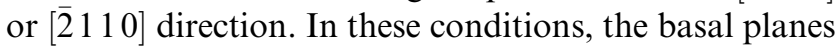
were set parallel to the incident beam direction. Dislocations in a basal slip system could then be easily distinguished from those in the non-basal slip systems. The straight dislocation segments lying parallel to the basal trace were in the basal-slip system. Otherwise, dislocations were in the non-basal slip systems.

Figs. 3(a) and (b) are two-beam images of an hcp grain $(\sim 160 \mu \mathrm{m}$ deep) taken with $(0002)$ and $(\overline{1} 2 \overline{1} 0)$ beam, respectively. Fig. 3(c) is the SAED pattern with

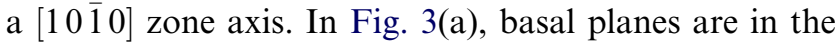
Bragg position $\left(\mathbf{g}=\left(\begin{array}{lll}0 & 0 & 2\end{array}\right)\right)$. With such an orientation, SFs are out of contrast. Apart from a few short segments (c-type dislocation), all dislocations are in basal planes. In Fig. 3(b), however, the SF fringes come onto contrast $(\mathbf{g} \cdot \mathbf{R} \neq 0$, integer $)$ and interfere with the dislocation images due to the distribution of SFs superimposed on the glide structure. It is, thus, impossible to uniquely identify a-type dislocations, since background fringes of SFs overlap on dislocation images in this reflection necessary for this purpose. However, strong interplay of SFs with dislocations is visible, indicative of the presence of a slip. Hence, the two-beam images with (0002) and (1210) spots, respectively, revealed pyramidal $(\mathbf{c}+\mathbf{a})$ and basal (a) slip character in the hep grain, but with low dislocation density.

\subsection{2. fcc phase}

3.1.2.1. Slip of dislocations. Fig. 4 is a cross-sectional image showing the intersecting planar arrays of dislocations in the fcc grain $(\sim 170 \mu \mathrm{m}$ deep $)$. Such dislocation pattern is characteristic of very low SFE fcc materials [30]. A decrease in SFE will result in more planar dislocation structure [31]. The formation of planar arrays of dislocations was observed to occur at depths ranging from $\sim 160$ to $180 \mu \mathrm{m}$. Thus, the slip of dislocations is considered to be the dominant strain accommodation in the fcc phase at the low strain level. This result was consistent with that occurring in the low SFE fcc alloys such as Inconel 600 alloy [11] and AISI 304 stainless steel [12] during the SMAT process.

\subsubsection{2. $\{111\}$ deformation twinning. With an increased} strain a transition of the mechanism for accommodating strain was observed. Fig. 5(a) is a bright-field TEM micrograph showing an fcc grain $(\sim 160 \mu \mathrm{m}$ deep). The thick plate-like twins (indicated by arrowheads) oriented in two $\{111\}$ planes are visible. They must form prior to deformation. At a high magnification (Fig. 5(b)), the
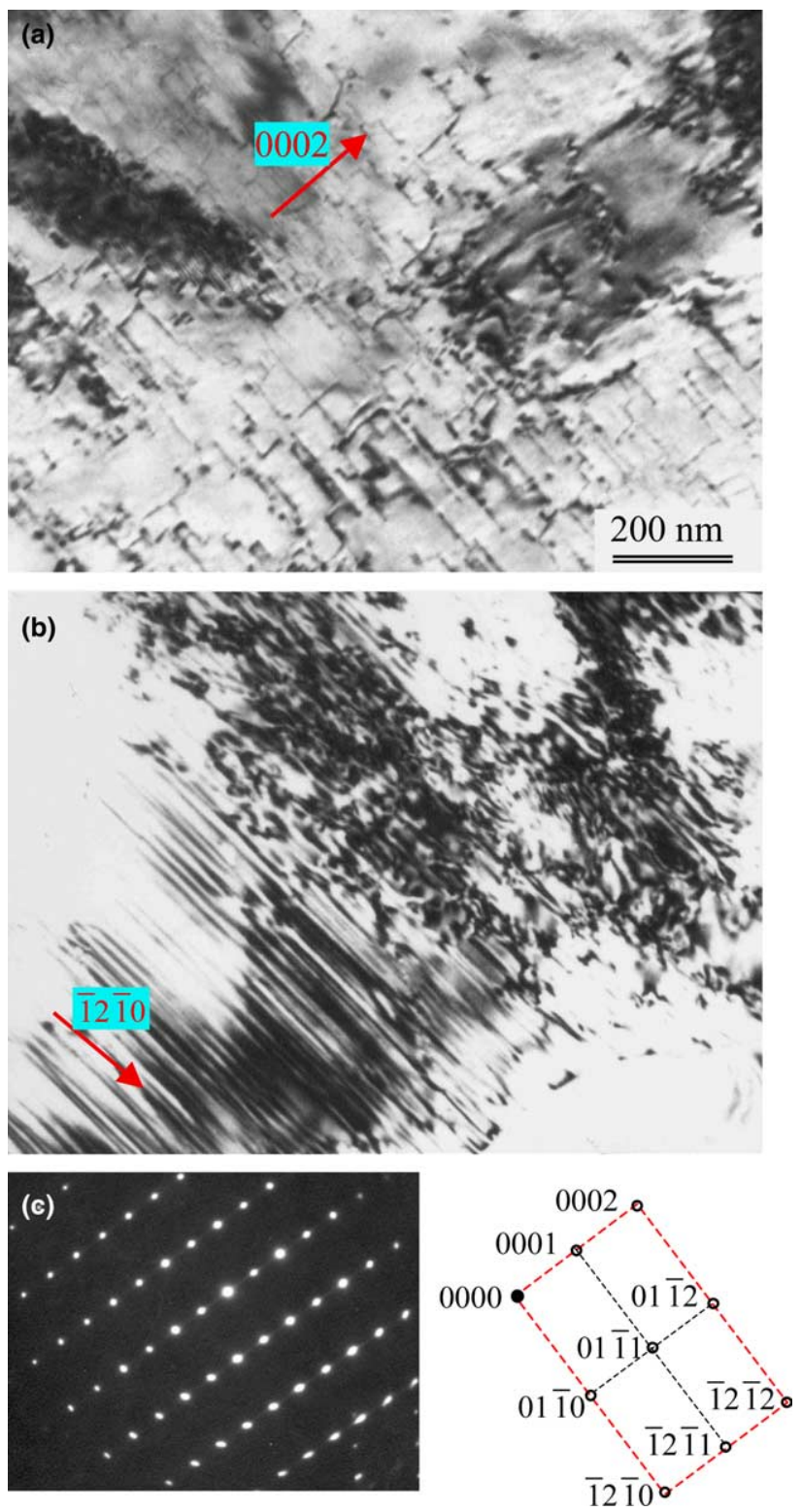

Fig. 3. Two-beam images of dislocation configurations in the hcp grain ( 160 $\mu \mathrm{m}$ deep): (a) (0002) image; (b) (1210) image; and (c) selected-area electron diffraction pattern with a zone axis $\left[\begin{array}{lll}1 & 0 & 1\end{array}\right]$.

presence of many very thin deformation twins developed with strain is seen. The SAED pattern in Fig. 5(c) indicates that they are $\{111\}$ deformation twins. A number of $\{111\}$ deformation twins in fcc grains were observed when deformation strain increases. This means the change of strain accommodation from slip of dislocations to twining occurring in the fcc phase. This change was also observed in Inconel alloy [11] and AISI 304 stainless steel [12] during the SMAT process.

3.1.2.3. $\gamma \rightarrow \varepsilon$ martensitic transformation. Figs. 6(a)-(d) are a set of TEM micrographs revealing the $\gamma \rightarrow \varepsilon$ martensitic transformation occurring in the fcc phase ( $\sim 150 \mu \mathrm{m}$ deep). Fig. $6(\mathrm{a})$ is the bright-field image show- 


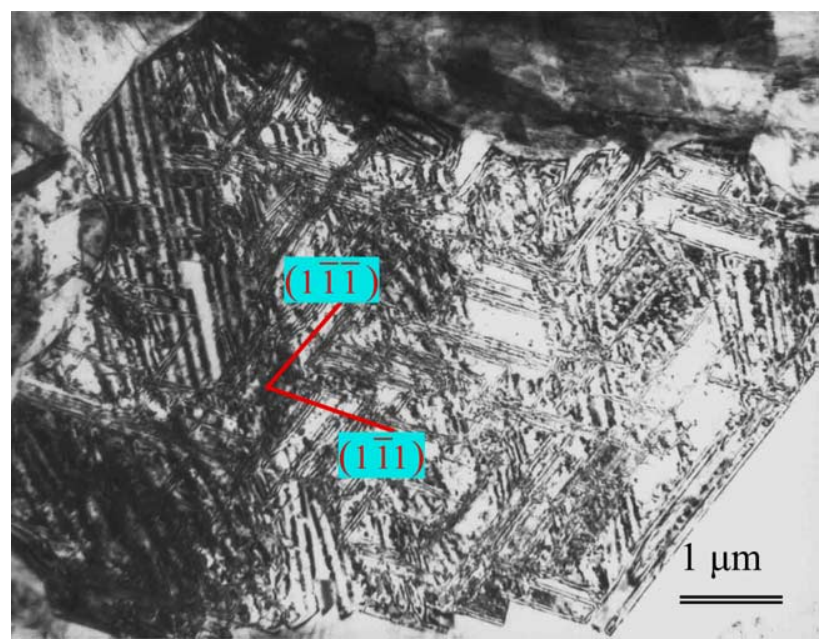

Fig. 4. The intersecting planar arrays of dislocations in the fcc grain ( $\sim 170 \mu \mathrm{m}$ deep).

ing the platelike feature of microstructure. From the SAED pattern in Fig. 6(e), the composite diffractions of $\gamma$-matrix, $\{111\}$ twin, and $\varepsilon$-martensite are identified,

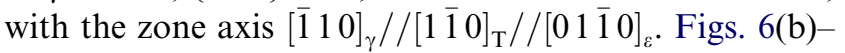
(d) are the dark-field images of $\gamma, \varepsilon$ and twins, respectively, corresponding to diffraction spots 1,2 and 3 in Fig. 6(e). Hence, the platelets in Fig. 6(a) consisted of the stacking of platelets of $\gamma$, twins, and $\varepsilon$, with intermediate regions of alternate twins and $\varepsilon$-martensites. It is particularly significant that the $\varepsilon$-martensite is recognized as hcp and is not to be associated with random-

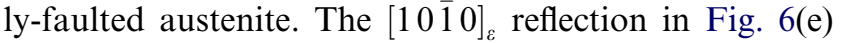
should not appear if the latter description applied [32]. The sharp $[0001]_{\varepsilon}$ reflection, which is forbidden by the structure factor but occurs through multiple diffraction, further indicates that the lattice is not randomly faulted. Long streaks are also associated with reflections since this phase is comprised of basal SFs (Fig. 6(c)) having limited thickness and normal to the plane of $(0001)_{\varepsilon}$. The martensitic transformation resulted in largely coherent hcp platelets, having the $(0001)$ habit plane and strictly complying with the following orientation

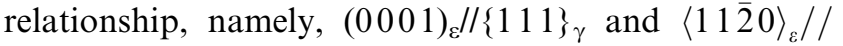
$\langle 110\rangle_{\gamma}$. This crystallographic relationship between $\varepsilon$ and $\gamma$ is the same as observed in cobalt [18], Multiphase cobalt alloy [33], and various stainless steels [34].

The $\gamma \rightarrow \varepsilon$ transformation continued with further increasing strain. Fig. 7 shows the intersecting network of $\varepsilon$-martensite platelets on three of four possible sets of $\{111\}_{\gamma}$ planes $(\sim 140 \mu \mathrm{m}$ deep $)$. The fourth set of $\{111\}_{\gamma}$ planes is parallel to the surface of the micrograph. The $\varepsilon$-martensite platelets of two orientations (indicated by $T_{1}$ and $T_{2}$, respectively) occur in areas between the first set of $\varepsilon$ platelets of $T_{3}$ orientation. Hence, the fcc phase will transform successively to $\varepsilon$-martensites via the $\gamma \rightarrow \varepsilon$ transformation with an increasing strain.
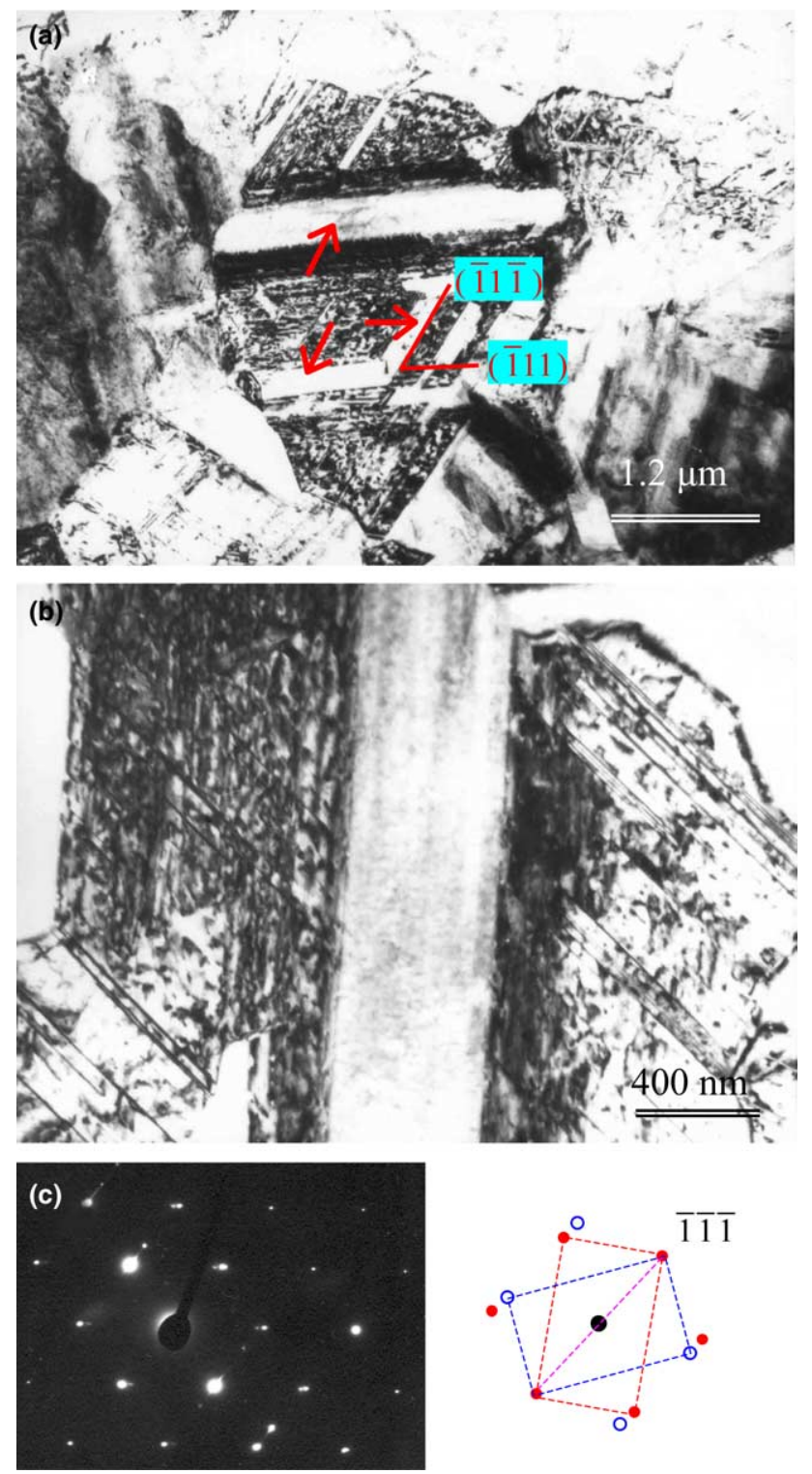

Fig. 5. TEM micrographs of deformation twins in the fcc grain (160 $\mu \mathrm{m}$ deep): (a) bright-field image; (b) high magnification of (a); and (c) selected-area electron diffraction pattern with a zone axis [1 10$]$.

\section{2. $80-130 \mu m$ below treated surface}

\subsubsection{Prismatic and basal slip}

With increasing strain level, the density of both $(\mathbf{c}+\mathbf{a})$ and a-type dislocations was observed to increase in the hcp grains, indicative of the presence of dominant slip of dislocations. Fig. 8(a) shows the formation of the low-angle dislocation subboundaries indicated by several pairs of arrowheads in an hcp grain $(\sim 110 \mu \mathrm{m}$ deep). These subboundaries are aligned approximately normal to the $\mathbf{g}$ vector of $\langle 10 \overline{1} 0\rangle$ and parallel to the [0002] direction. This indicates that the slip of dislocations occurring on the $\{1010\}$ prism planes [13]. Because the slip of dislocation in hep cobalt was conclusively determined to be 

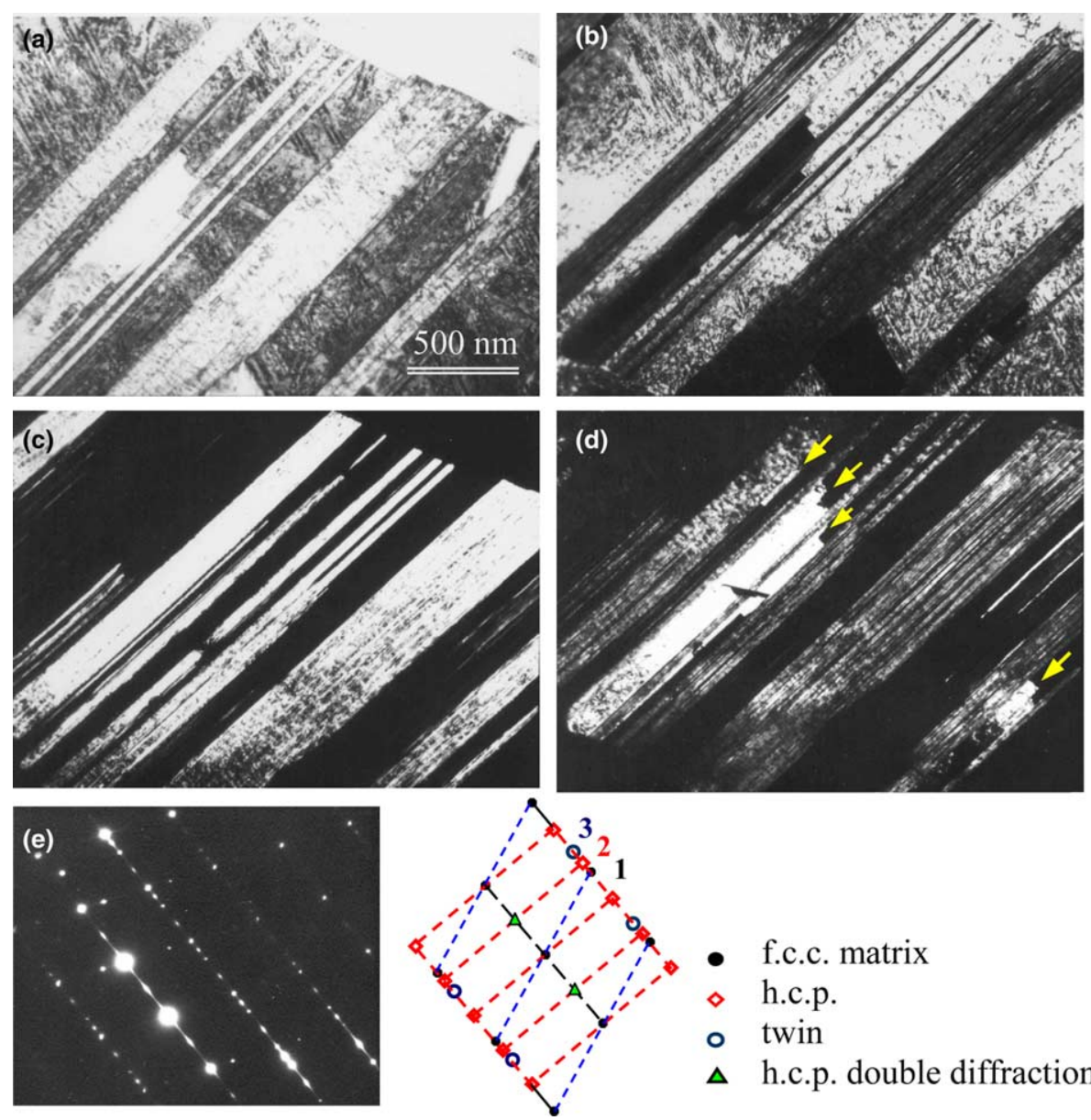

- f.c.c. matrix

$\diamond$ h.c.p.

o twin

$\Delta$ h.c.p. double diffraction

Fig. 6. TEM micrographs of the microstructure showing the $\gamma \rightarrow \varepsilon$ martensitic transformation occurring in the fcc grain $(\sim 150 \mu \mathrm{m}$ deep): (a) brightfield image; (b)-(d) dark-field images of $\gamma, \varepsilon$-martensite and twin (indicated by arrowheads); and (e) selected-area electron diffraction pattern with

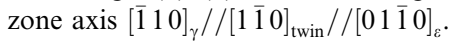

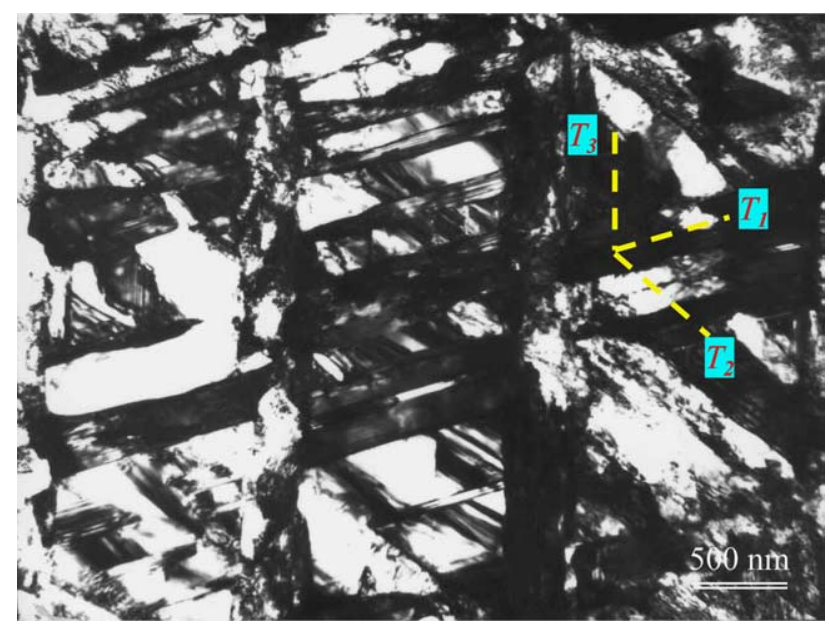

Fig. 7. The intersecting network of $\varepsilon$-martensite platelets on three sets of $\{111\}_{\gamma}$ planes $(\sim 140 \mu \mathrm{m}$ deep).

$\langle 11 \overline{2} 0\rangle[35,36]$, the prism slip system in the present study should be $\langle 11 \overline{2} 0\rangle\{10 \overline{1} 0\}$. Fig. 8(b) shows the presence of dislocation walls (indicated by two pairs of arrowheads), with their orientations normal to the $\langle 10 \overline{1} 0\rangle$ direction. These dislocation walls will much likely evolve into dislocation subboundaries at increased strain, as observed extensively in cubic metals during deformation [6-9].

Fig. 9(a) exhibits the presence of subgrains arising from dislocation subboundaries (indicted by several pairs of arrowheads) in an hcp grain. The subboundaries are normal to SF fringes, indicative of $\langle 11 \overline{2} 0\rangle\{1 \overline{1} 00\}$ prism slip. Figs. 9(b) and (c) are images obtained by tilting the foil to a few degrees. Some subgrains are out of contrast due to the presence of misorientations between them. It is of interest to note that these subgrains also have the additional subboundaries parallel to SF fringes, i.e., normal to the $\mathbf{g}$ vector of $\langle 0002\rangle$. They should result from slip of dislocations on basal planes in the $\langle 11 \overline{2} 0\rangle$ direction. Hence, the co-operation of both $\langle 11 \overline{2} 0\rangle(0001)$ basal and $\langle 11 \overline{2} 0\rangle\{10 \overline{1} 0\}$ prism slip causes the subgrain formation and subsequent increase in misorientations of subgrains. The subboundaries due to basal slip are, in fact, difficult to be 'seen' due 


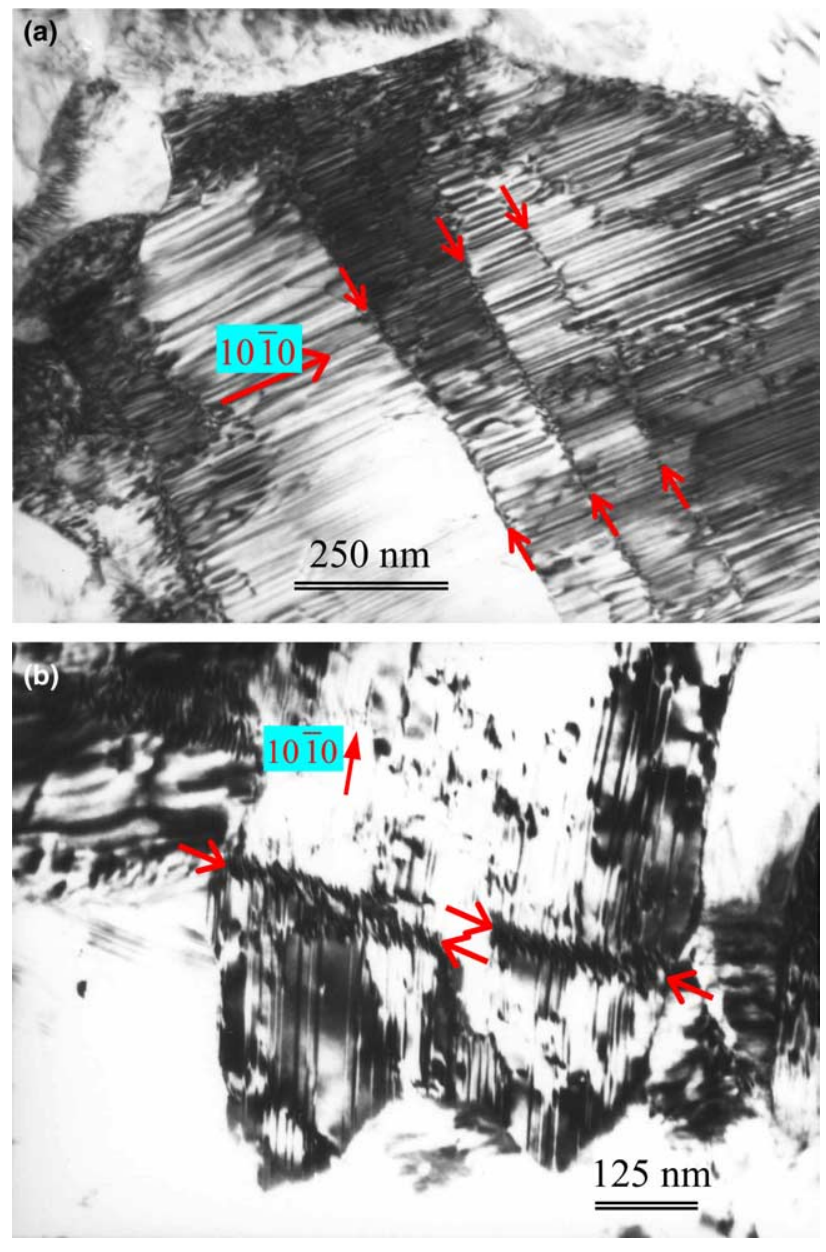

Fig. 8. TEM micrographs of (a) low-angle dislocation boundaries and (b) dislocation walls in the hcp grain (indicated by several pairs of arrowheads) ( 110 $\mu$ m deep).

to overlapping of background fringes of SFs with dislocation images.

\subsubsection{Grain subdivision}

Fig. 10(a) reveals a typical example of grain refinement occurring in an hcp grain. A large number of refined small grains are present inside the original large grain. It is of interest to note the presence of the SF fringes of various orientations in most small grains. Some low angle dislocation subboundaries (indicated by triangles) normal to the SF fringes are visible, indicative of the operation of $\langle 11 \overline{2} 0\rangle\{10 \overline{1} 0\}$ prism slip. Hence, the grain refinement takes place through subdivision mechanism due to both prismatic and basal slip. Fig. 10(b) is the SAED pattern taken from the original large grain, indicating high misorientations among refined grains.

\subsection{0-80 $\mu \mathrm{m}$ below treated surface}

With successive grain subdivision, the process of grain refinement continued and hence, the ultrafine crys-
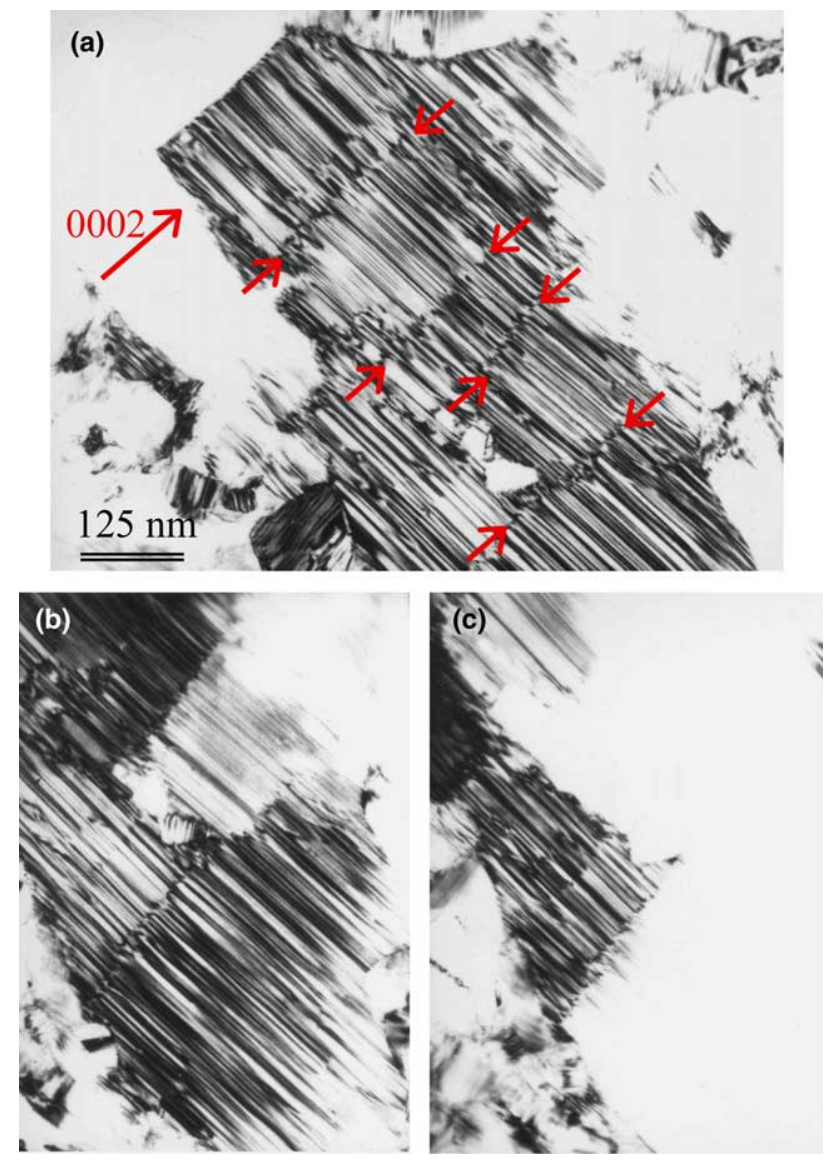

Fig. 9. TEM micrographs of an hcp grain $(\sim 110 \mu \mathrm{m}$ deep): (a) subgrains (note the presence of dislocation boundaries indicated by several pairs of arrowheads); (b) and (c) misorientations of subgrains by tilting the foil to a few degree.

talline grains formed. Fig. 11 shows the microstructure of ultrafine crystallites $(\sim 70 \mu \mathrm{m}$ deep $)$. It is seen again that the SFs appear in most grains.

\subsection{5-40 $\mu m$ below treated surface}

The deformation strain and strain rate are drastically increased in the top surface layer [27], leading to formation of nc grains. Fig. 12(a) shows the microstructure $(\sim 40 \mu \mathrm{m}$ deep) where most grains are smaller than $100 \mathrm{~nm}$. The SAED pattern in Fig. 12(c) consists of discontinuous rings, with a number of strong spots diffused at an angle ranging from $15^{\circ}$ to $25^{\circ}$. Although characteristic mirror-type diffraction spots from a twin system are not evident, strong (10 101 ) twin spots are visible, indicating that the viewing area of the SAED previously was of twin relation. In fact, a boundary (indicated by several arrowheads) nearly normal to $(10 \overline{1} 1)$ plane can be seen in Fig. 12(a). Fig. 12(b) shows the nc grains of $\sim 20$ $\mu \mathrm{m}$ deep, with a mean grain size $\sim 50 \mathrm{~nm}$. The SAED pattern in Fig. 12(d) consists of a set of sharp rings, 

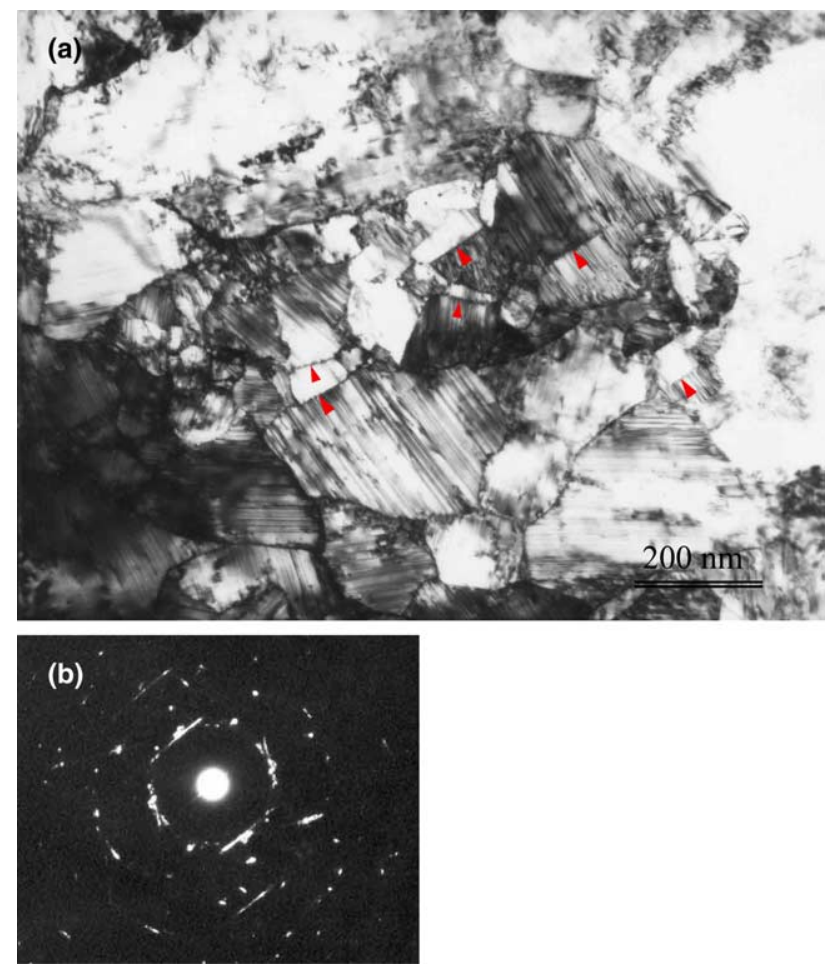

Fig. 10. (a) TEM micrograph of the microstructure $(\sim 100 \mu \mathrm{m}$ deep) showing presence of a large number of refined grains. (b) selected-area electron diffraction pattern taken from the original large grain.

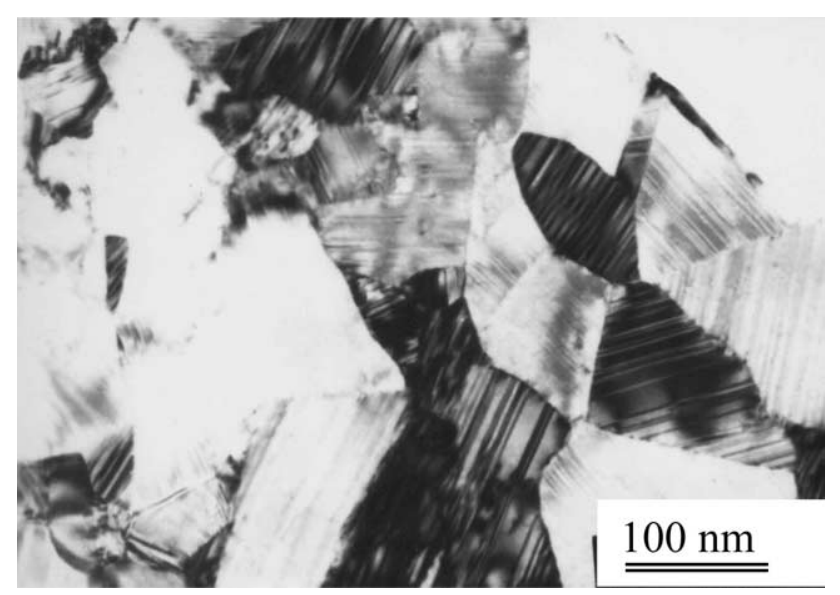

Fig. 11. The ultrafine crystallites $(\sim 70 \mu \mathrm{m}$ deep).

indicating high misorientations among most of grains compared with that in (a).

\subsection{Formation of $n c$ grains at grain boundary and triple junction}

Accompanying the process of grain refinement through slip-induced subdivision, a special phenomenon of nc formation is also observed. Fig. 13 shows the development of nc grains directly at the grain boundaries and triple junctions $(\sim 60 \mu \mathrm{m}$ deep). The
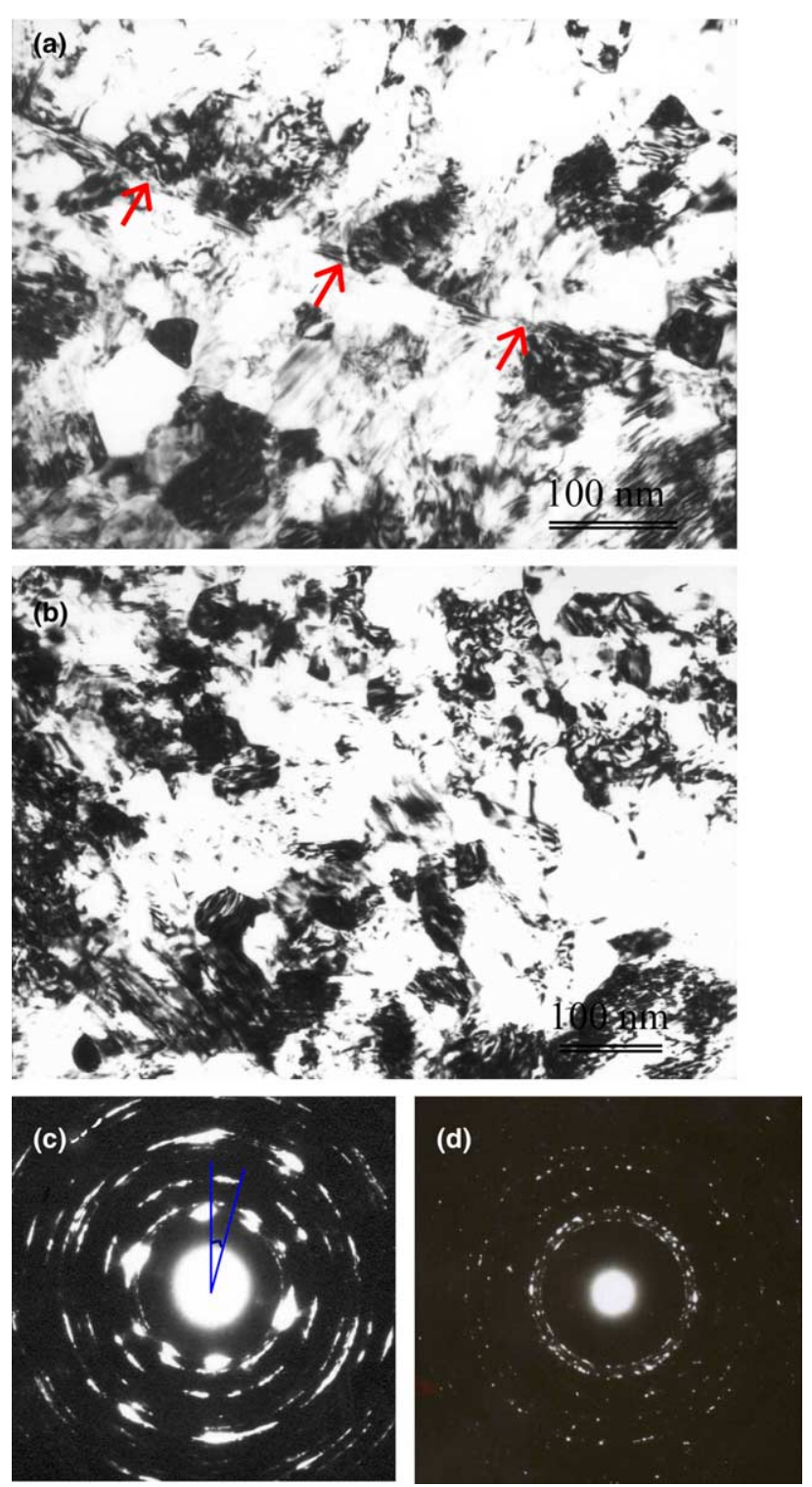

Fig. 12. TEM micrographs of nanocrystalline grains of (a) $\sim 40 \mu \mathrm{m}$ and (b) $20 \mu \mathrm{m}$ deep, with corresponding respective selected-area electron diffraction patterns (c) and (d).

grain sizes of nc grains range from $\sim 10$ to $100 \mathrm{~nm}$. In particular, these grains have SFs in their interior even at their initial stage of formation. This phenomenon of nc formation was observed to concurrently occur with grain subdivision.

\section{Discussion}

Based on TEM observations, the entire process of microstructural evolution occurring at various depths of the deformed surface layer is summarized in Table 1 . The process of grain refinement will be discussed in terms of strain accommodation in both hep and fcc phases at different levels of strain. 


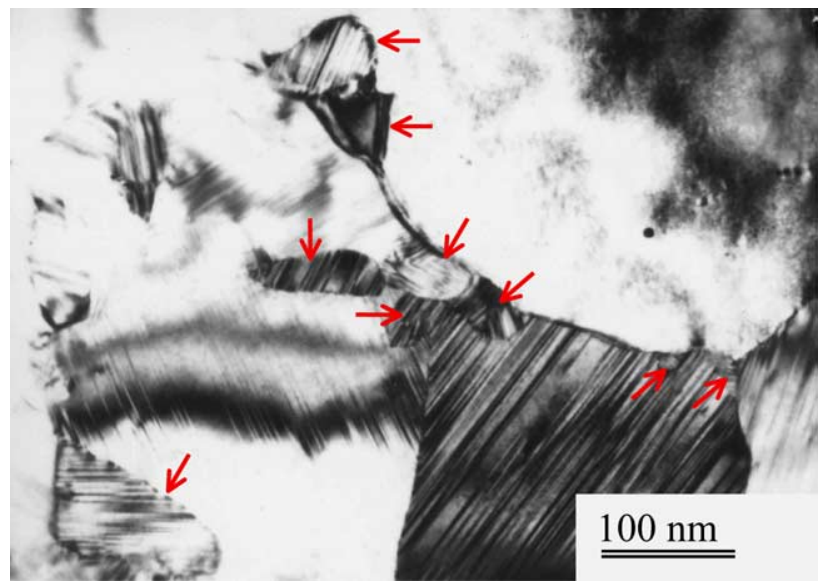

Fig. 13. The formation of nc grains indicated by arrowheads at the grain boundary and triple junction $(\sim 60 \mu \mathrm{m}$ deep).

Table 1

Summary of microstructural evolution in the deformed surface layer at various depths

\begin{tabular}{lll}
\hline $\begin{array}{l}\text { Depth below } \\
\text { surface }(\mu \mathrm{m})\end{array}$ & hcp phase & fcc phase \\
\hline $15-40$ & $\begin{array}{l}\text { Nanocrystallite formation } \\
\text { Grain subdivision } \\
40-80\end{array}$ & \\
& $\begin{array}{l}\text { Nanocrystallite nucleation } \\
\text { at grain boundary and } \\
\text { triple junction }\end{array}$ & \\
& $\begin{array}{l}\text { Basal and prism slip } \\
\text { Increased dislocation density }\end{array}$ & \\
$80-130$ & $\begin{array}{l}\{10 \overline{1} 1\} \text { Twinning } \\
\text { Basal stacking faults } \\
\text { a and }(\mathbf{a}+\mathbf{c}) \text { dislocations } \\
\text { Equiaxed grains with SFs }\end{array}$ & $\begin{array}{l}\text { Slip of dislocations } \\
\{111\} \text { Twinning } \\
\text { Equiaxed grains with } \\
\text { twins and SFs }\end{array}$ \\
\hline
\end{tabular}

\subsection{Grain refinement of hcp phase}

At the low strain level during the SMAT process, the imposed strain is accommodated mainly by $\{10 \overline{1} 1\}$ deformation twinning in the hep phase as shown in Fig. 1, rather than by slip of dislocations as is often found in cubic metals [6-10]. The $\{10 \overline{1} 1\}$ twinning was not reported in the previous literature during plastic deformation of poly- and single-crystal cobalt. Thus, further detailed study of the origin of this behavior is warranted. The $\{10 \overline{1} 1\}$ twinning of "b4" mode with shear strain of only 0.1 [29] probably operates, due to twinning occurring at the low strain level. $\{10 \overline{1} 1\}$ twinning was also observed to be the main strain accommodation in titanium during the first ECAP pass with shear strain $\sim 1.83$ [13] and, the "b3" or "b1" type $\{10 \overline{1} 1\}$ twinning, with shear strain 0.55 and 1.44 , respectively, was proposed to operate [13].

The deformed hcp grains contain (0001) basal SFs. They exist even in nc grains (Figs. 11-13). The forma- tion of SFs involves the partial dislocation activity and hence, will have a significant effect on deformation behavior especially at the nanometer scale [37,38]. In addition, both pyramidal $(\mathbf{a}+\mathbf{c})$ and basal (a) dislocations are observed in the hcp phase during plastic deformation.

The prismatic $\langle 11 \overline{2} 0\rangle\{1 \overline{1} 00\}$ and basal $\langle 11 \overline{2} 0\rangle$ (0001) slip starts to operate with increased strain as seen in Figs. 8 and 9. The operative slip systems in hcp metals are strongly dependent on the axial ratio $(c / a)$. With a $c / a$ ratio less than the ideal value (i.e., $<1.633$ ), the lattice resistance for prism planes is lower than that for basal and pyramidal planes and, hence, slip occurs easily on prism planes [39]. In cobalt with a $c / a$ ratio close to the ideal value, both prism slip and basal slip have been identified as primary slip systems $[35,40,41]$. The present study suggests that prism and basal slip become dominant mechanism of accommodating strain with increased strain during the SMAT process. The grain refinement must have increased the critical resolved shear stress (CRSS) for twinning over that for slip of dislocations. The grain size dependence of the CRSS for twinning has been described in the hop structure $[42,43]$. The rate of increase in the twinning stress should be much larger than that of dislocation slip as the grain size decreases. The effective grain size decreases due to the fact that twin boundaries are reported to act as obstacles for slip and dislocations may be blocked by twin boundaries $[44,45]$. Therefore, the CRSS for twinning increases more significantly compared with that of slip as the effective grain size decreases, promoting the deformation by dislocation slip with increased strain. The change of strain accommodation from deformation twinning to dislocation slip is also observed in other hcp metals, such as titanium [13,14], magnesium [15] and zirconium [16].

The grain refinement via grain subdivision takes place due to the onset of both prism and basal slip. The slip causes the formation of low-angle dislocation subboundaries surrounding subgrains as indicated in Figs. 8-10. Simultaneously, the misorientations of subgrains increase with strain. The process of grain subdivision may proceed successively to a finer and finer scale with strain, resulting in the formation of ultrafine crystallites and nanocrystallites. Hence, the present grain subdivision through the slip of dislocations seems to exhibit the typical character much similar to that occurring in cubic metals [6-9]. The highly misoriented and random grain boundaries will then result with increasing strain. The grain rotation and grain boundary sliding should be responsible for the random grain boundary of $\mathrm{nc}$ grains $[46,47]$.

The presence of nanocrystallites at the grain boundaries and triple junctions, as shown in Fig. 13, undoubtedly indicates a unique phenomenon of nc formation. This phenomenon may be understood in terms of a 
dynamic recrystallization (DRX) mechanism. Zhang et al. [48] have studied the nc formation in hep zinc, which was prepared from cryomilled powders. They observed a bimodal grain size distribution in the early stage of cryomilling and explained the formation of large numbers of small nc grains $(\sim 2-6 \mathrm{~nm})$ in terms of a DRX mechanism. They considered that cryomilling may induce much high strain into the deformed metals, thus creating some highly strained regions. These areas may have stored enough energy to allow the nucleation of small nanocrystallites to occur during the cryomilling process. With high levels of dislocation density, small energy fluctuations resulting from impacts during the cryomilling process can trigger a DRX event. The nucleation should happen especially in certain deformation inhomogeneities like shear bands, grain boundaries and triple junctions. Grain boundaries and triple junctions are locations of inhomogeneous deformation because of the compatibility strain that is necessary to accommodate an incongruent shape change of grains at their common grain boundaries, or if grain boundary sliding is obstructed. This makes them particularly liable to nucleation of DRX. In essence, grain boundaries and triple junctions are the most likely nucleation sites for DRX in polycrystals. In addition, the temperature in the treated layer will rise due to repetitive peening of balls during the SMAT process and hence, the onset of a DRX event is easy to happen. Furthermore, Zhang et al. [48] also found that the typical grain size refinement mechanism is operative in terms of the decomposition of large grains during milling. Two mechanisms may operate simultaneously. This is in line with the present investigation.

\subsection{Grain refinement of $f c c$ phase}

The dislocation slip is observed to accommodate strain in the fcc grain of cobalt at the low strain level (Fig. 4). The slip of dislocations occurs on their respective $\{111\}$ planes, forming intersecting planar arrays of dislocations, typical of dislocation configurations observed in the fcc structure with a low SFE at low strain. The dislocation cells, dislocation walls and dislocation tangles are not observed as often happened in cubic materials [6-10]. With increased strain, fcc grains experience $\{111\}$ deformation twinning as the primary plastic deformation mechanism (Fig. 5). The change of accommodating strain from dislocation slip to twinning in the present investigation was consistent with the results reported in low SFE fcc materials $[11,12,45]$. With further increasing strain, the $\gamma \rightarrow \varepsilon$ martensitic transformation occurs in the fcc phase, as shown in Figs. 6 and 7. The $\gamma \rightarrow \varepsilon$ transformation, in essence, contributes grain refinement in two respects. First, the transformation results in the formation of refined $\varepsilon$ platelets and second, the $\varepsilon$ platelets can be thought of as grain boundaries inducing a refinement effect by subdividing the grains with the same function as deformation twins.

\section{Summary}

A comprehensive experimental investigation has been performed to document the grain refinement and strain accommodation in cobalt (a mixture of hep phase together with fcc phase) subjected to the surface mechanical attrition treatment. Specific conclusions are drawn as follows:

1. The hep phase accommodated plastic deformation by $\{10 \overline{1} 1\}$ deformation twinning at low strain level. The hcp phase contained the high density basal stacking faults and basal (a) and pyramidal $(\mathbf{a}+\mathbf{c})$ dislocations. With increasing strain, the strain accommodation in the hcp phase transformed to $\langle 11 \overline{2} 0\rangle\{1 \overline{1} 00\}$ prism and $\langle 11 \overline{2} 0\rangle(0001)$ basal slip, leading to formation of low angle dislocation boundaries surrounding subgrains. The grain subdivision due to slip of dislocations became the dominant mechanism of grain refinement and as a result, ultrafine crystallites and nanocrystallites are formed. Moreover, the nc grains may nucleate directly at the grain boundaries and triple junctions. Their formation may be produced by a dynamic recrystallization mechanism.

2. The strain accommodation in the fcc phase started by the slip of dislocations at low strain, forming intersecting planar arrays of dislocations. With increasing strain, $\{111\}$ deformation twinning was activated as a primary deformation mechanism. With further increased strain level, the $\gamma \rightarrow \varepsilon$ transformation occurred with following orientation relationship: $(0001)_{\varepsilon} / /\{111\}_{\gamma}$ and $\langle 11 \overline{2} 0\rangle_{\varepsilon} / /\langle 110\rangle_{\gamma}$. The hcp platelets developed on the $\{111\}$ planes of the fcc phase, forming in most cases an intersecting network. The $\gamma \rightarrow \varepsilon$ transformation contributed to grain refinement.

\section{Acknowledgements}

This research was supported by National Basic Research Program of China through Grant No. 2004CB619305, National Natural Science Foundation of China (50471086, 10472117, 50021101), The Chinese Academy of Sciences, NEDO International Joint Research Grant Program (01MB5), and Ministry of Research of French and the Regional Council of Champagne Ardenne France (Grant 2001882, CPER EN 2040). 


\section{References}

[1] Kumar KS, van Swygenhoven H, Suresh S. Acta Mater 2003;51:5743.

[2] Lu L, Sui ML, Lu K. Science 2000;287:1463.

[3] Chen MW, Ma E, Hemker KJ, Sheng HW, Wang YM. Science 2003;300:1275.

[4] Tong WP, Tao NR, Wang ZB, Lu J, Lu K. Science 2003;299:686.

[5] Valiev RZ. Nat Mater 2004;3:511.

[6] Hughes DA, Hansen N. Phy Rev Lett 2001;87:135503.

[7] Huang JY, Zhu YT, Jiang H, Lowe TC. Acta Mater 2001;49:1497.

[8] Wu X, Tao N, Hong Y, Xu B, Lu J, Lu K. Acta Mater 2002;50:2075.

[9] Tao NR, Wang ZB, Tong WP, Sui ML, Lu J, Lu K. Acta Mater 2002;50:4603.

[10] Iwahashi Y, Horita Z, Nemoto M, Langdon TG. Acta Mater 1997;45:4733.

[11] Tao NR, Wu X, Sui ML, Lu J, Lu K. J Mater Res 2004;19:1623.

[12] Zhang HW, Hei ZK, Liu G, Lu J, Lu K. Acta Mater 2003;51:1871.

[13] Shin DH, Kim I, Kim J, Kim YS, Semiatin SL. Acta Mater 2003;51:983.

[14] Zhu KY, Vassel A, Brisset F, Lu K, Lu J. Acta Mater 2004;52:4101.

[15] Kaibyshev R, Sitdikov O. Z Metallkd 1994;85:738.

[16] Choi WS, Ryoo HS, Hwang SK, Kim MH, Swun SI, Chae SW. Metall Mater Trans A 2002;33A:973.

[17] Korner A, Karnthaler HP. Philos Mag A 1983;48:469.

[18] Christian JW. The theory of transformations in metals and alloys. New York: Pergamon Press; 1965. p. 808-11.

[19] Bibby MJ, Gordon Parr J. Cobalt 1963;20:111.

[20] Hitzenberger C, Karnthaler HP, Korner A. Phys Stat Sol (a) 1985;89:133.

[21] Eppelsheimer DS, Wilcox RC. J Inst Metals 1955;93:229.

[22] Seeger S, Kronmuller H, Boser O, Rapp M. Phys Stat Sol 1963;3:1107.
[23] Vaidya S, Mahajan S. Acta Metall 1980;28:1123.

[24] Sort J, Zhilyaev A, Zielinska M, Nogues J, Surinach S, Thibault J, et al. Acta Mater 2003;51:6385.

[25] Huang JY, Wu YQ, Ye HQ. Appl Phys Lett 1995;66:308.

[26] Karimpoor AA, Erb U, Aust KT, Palumbo G. Scripta Mater 2003;49:651.

[27] Lu K, Lu J. Mater Sci Eng A 2004;375-377:38.

[28] Bibring H, Sebilleau F, Buckle CJ. Inst Metals 1958-59;87:71.

[29] Paton NE, Backofen WA. Metall Trans 1970;1:2839.

[30] Singh RP, Doherty RD. Metall Trans A 1992;23A:307.

[31] Hull D, Bacon DJ. Dislocations. New York: Oxford University Press; 1989

[32] Venables JA. Phil Mag 1962;7:35.

[33] Drapier JM, Viatour P, Coutsouradis D, Harbraken L. Cobalt 1970;49:171.

[34] Remy L, Pineau A. Metall Trans 1974;5:963.

[35] Yoo MH, Wei CT. J App Phys 1967;38:4317.

[36] Chin GY, Mammel WL. Metall Trans 1970;1:357.

[37] van Swygenhoven H, Derlet PM, Hasnaoui A. Phys Rev B 2002;66:024101.

[38] Yamakov V, Wolf D, Phillpot SR, Mukherjee AK, Gleiter H. Nat Mater 2002;1:45.

[39] FrostH,AshbyM.Deformation-mechanismmaps. Oxford: Pergamon Press; 1982.

[40] Kopetskiy CV, Myshlyayev MM, Novokhatskaya NI, Yukhanov VA. Phys Met Metallogr 1975;39:164.

[41] Churchman AT. Trans TMS-AIME 1960;218:262.

[42] Madhava NM, Worthington PJ, Armstrong RW. Phil Mag 1972;25:519.

[43] Mayer MA, Vohringer D, Lubarda VA. Acta Mater 2001;49:4025.

[44] Lu L, Shen YF, Chen XH, Qian LH, Lu K. Science 2004;304:422.

[45] Karaman I, Sehitoglu H, Chumlyakov, Maier HJ. JOM 2002;31.

[46] Ovid'ko IA. Science 2002;295:2386.

[47] van Swygenhoven H, Farkas D, Caro A. Phys Rev B 2000;62:831.

[48] Zhang X, Wang H, Narayan J, Koch CC. Acta Mater 2001;49:1319. 